\title{
The Angiotensin AT2 Receptor: A major constituent of the "Protective Arm" of the Renin-Angiotensin System
}

\section{Thomas Unger \\ Em. Professor of Pharmacology and Experimental Medicine CARIM - Maastricht University, The Netherlands}

\section{Abstract}

Since its discovery, more than twenty-five years ago, the angiotensin AT2 receptor (AT2R) has puzzled the scientific community because of its distinct localization, regulation, signalling pathways and biological effects separating it clearly from the classical features of the renin-angiotensin system (RAS) mediated by the angiotensin AT1 receptor. Intensive research over the years has revealed major characteristics of the AT2R as a modulatory player involved in anti-proliferation, anti-inflammation, natriuresis, neuroregeneration and apoptosis, i.e. biological programs that can counterbalance patholological processes and enable recovery from disease. The AT2R has thus mutated from an "enigmatic" receptor to a significant member of the "protective arm" of the RAS. The recent development of novel, small molecule-and peptide-derived AT2R agonists offers a therapeutic potential in humans with a variety of clinical indications.

The 'enigmatic receptor' is a designation of the angiotensin AT2 receptor (AT2R) from the very beginning of its discovery more than 25 years ago in the late 1980s. 'Enigmatic' because of constitutive action, atypical intracellular signalling and "hidden" (patho)physiological functions that took years, almost decades, to unveil. Even today the various actions, some of them still controversial, the numerous intracellular signalling pathways, the interaction with other membrane receptors and the role of the AT2R in the context of the renin-angiotensin system (RAS), are far from being fully elucidated. We at least know much more than in the early days about the AT2R, we can confidently classify it as an important member of the "protective arm " of the RAS (Figure 1) and we have even identified this receptor as a therapeutic target.

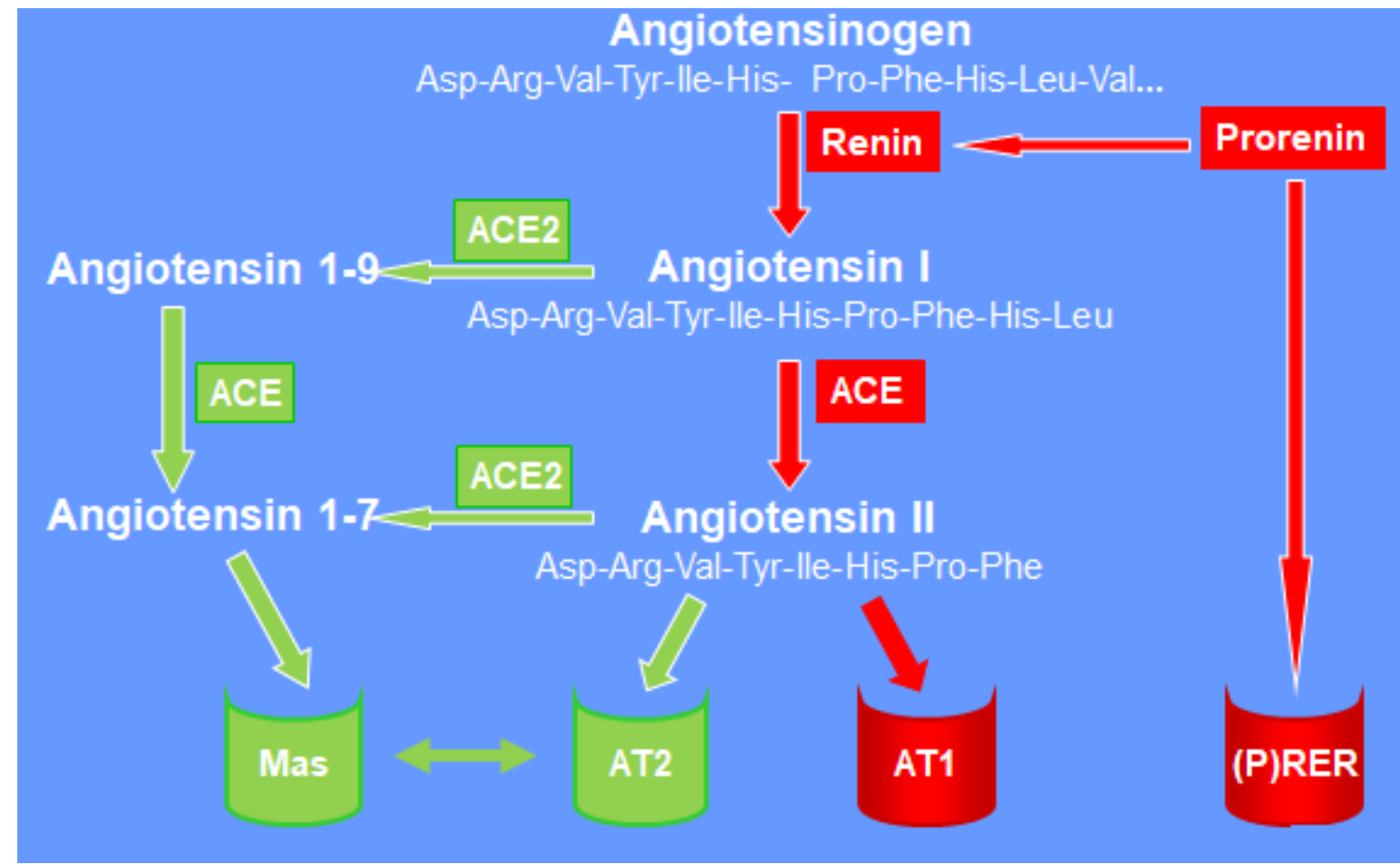

Figure 1

The Renin-Angiotensin System with its „classical" arms (in red) and its "protective arms" (in green)

Continued overleaf 
Until about 1989, the scientific community thought that angiotensin II (Ang II), the main effector peptide of the RAS, used only one single receptor to exert its various actions in the cardiovascular system and beyond: the angiotensin receptor. Several peptidergic angiotensin derivatives such as saralasin, had been developed to antagonize those Ang II actions thought to be harmful or just to serve as pharmaceutical tools to gain more insight into the role of the RAS. However, for several reasons, expectations as to developing these compounds into clinically useful antihypertensives could not be fulfilled.

Around the late 80 s, some pharmaceutical companies, searching for new tools and better drugs interfering with the RAS, had developed small compounds that were differentially binding to angiotensin receptors in various tissues pointing to distinct angiotensin receptor populations. While the "sartans" (ARBs) selectively bound to the "classical" angiotensin receptor in blood vessels and other tissues later designated as the angiotensin AT1 receptor (AT1R), some other agents were surprisingly binding to a "new" angiotensin receptor in uterus and adrenal gland, later designed as the angiotensin AT2 receptor (AT2R).

Retrospectively, the surprise about two or even more receptor subtypes in one biological system, such as the RAS, seems to be somewhat out of place since many of these systems operate on several receptors and the effects mediated by different receptors in these systems are often different from- or even opposing each other.

Unexpected pharmacological binding does not necessarily prove the existence of a "new" receptor. Proof of the existence of the AT2R was first provided by the molecular cloning of CDNA of this receptor by Victor Dzau's laboratory at Stanford and Tadashi Inagami's group at Vanderbilt. With further characterization of the genomic structure and the documentation of mouse phenotype by gene deletion experiments, the AT2R was no longer a pharmacologic binding phenomenon but a real biologic entity.

In the following years several groups, including our own, provided ample evidence based on different experimental approaches that Ang II via its AT2R induced effects such as anti-proliferation or anti-inflammation quite opposite to those "classical" actions of the Renin-Angiotensin

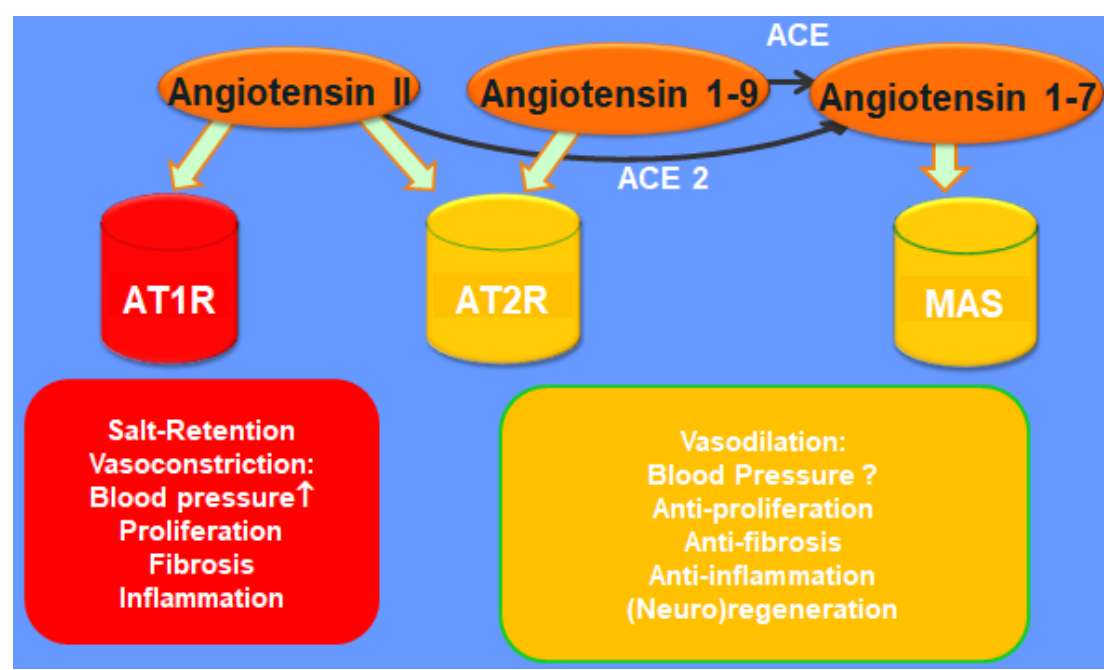

Figure 2 Effects of the two arms of the Renin-Angiotensin System.

System, exerted via its AT1R.

The international research community did not readily accept findings against a mantra of the time stating that Ang II was exclusively acting as a proliferative / hypertrophic agent in cells of the cardiovascular system. It took some more years until it was generally accepted that Ang II, via its AT2R, could act indeed as an growth-inhibiting principle, assigning for the first time a clear biological function to the "enigmatic" AT2R: anti-proliferation with a predominant localization in, among others, fetal tissue, the uterus and some distinct areas of the brain.

It also became apparent that AT2R signalling differed markedly from AT1R signalling. While both receptors, though having only 33-34\% homology, could be assigned to the seven transmembrane domain family of receptors which usually bind G-proteins, the AT2R exhibited some atypical features. It engages a complex intracellular signalling network linked to distinct physiological functions: anti-proliferation (but under some special conditions also hypertrophy),

anti-inflammation, cellular differentiation, anti-fibrosis, natriuresis, and induction or inhibition of apoptosis (Figure 2).

These findings fuelled a vivid discussion, sometimes controversy, about the "true" biological functions of the AT2R. More recently a model to clear up several contradictions has been proposed in which the type of adapter protein recruited to the receptor, as well as the presence or absence of growth factors, determine the cellular effects assuming a multiple-state receptor model with several activated states. Such a "biased agonism" model could also be the basis for our mechanistic understanding of compounds selectively stimulating the AT2R. In addition, different patterns of homo- or heterodimerization (with the AT1R, the MAS receptor or the bradykinin $B 2$ receptor) have to be considered as functional determinants of AT2R action. It also became apparent that this receptor is distinctly regulated: while being highly expressed in fetal tissues but suppressed in many tissues in the adult organism, its expression can be drastically upregulated under the condition of ischemic or traumatic tissue injury but also in atherosclerotic lesions. 
A further aspect of AT2R signalling and function deserves particular attention, a feature quite novel and unique for the RAS: Neuroprotection and neuroregeneration, modulation of sympatho-excitation, and possibly improvement of cognitive function. Different experimental approaches used by independent groups revealed a fairly consistent picture of the AT2R in the nervous system, revealing that the AT2R-induced neuronal differentiation and inhibition of neuro-excitation could be not only part of neuronal development but also the basis for therapeutical considerations about drug-induced AT2R stimulation. This idea was supported later-on with the help of a wealth of results in different experimental disease models.

Together all these experimental results point to a possible qualification of the AT2R as a drug target. A decisive step forward in this direction came in 2004 with the publication of the first highly selective, orally active AT2R agonist by a Swedish group. Until now compound 21, the main representative of this family of AT2R agonists, has not only helped to unravel many of the secrets of $A T 2 R$ signalling and function but, moreover and most importantly, has opened the door to numerous potential clinical indications of AT2R stimulation not only in the cardiovasular field but also in the areas of renal, metabolic and neuronal diseases as well as in many other indication areas where anti-inflammatory, antifibrotic or anti-proliferative actions are therapeutically required.

One more interesting feature is that despite the fact that activation of the AT2R can engender the production of cGMP via the nitric oxide (NO) pathway, AT2R stimulators do not seem to be direct antihypertensive agents such as the AT1R antagonists. However, they could contribute to lowering blood pressure via indirect mechanisms, for instance by their antifibrotic actions reducing arterial stiffness (Figure 3 ).

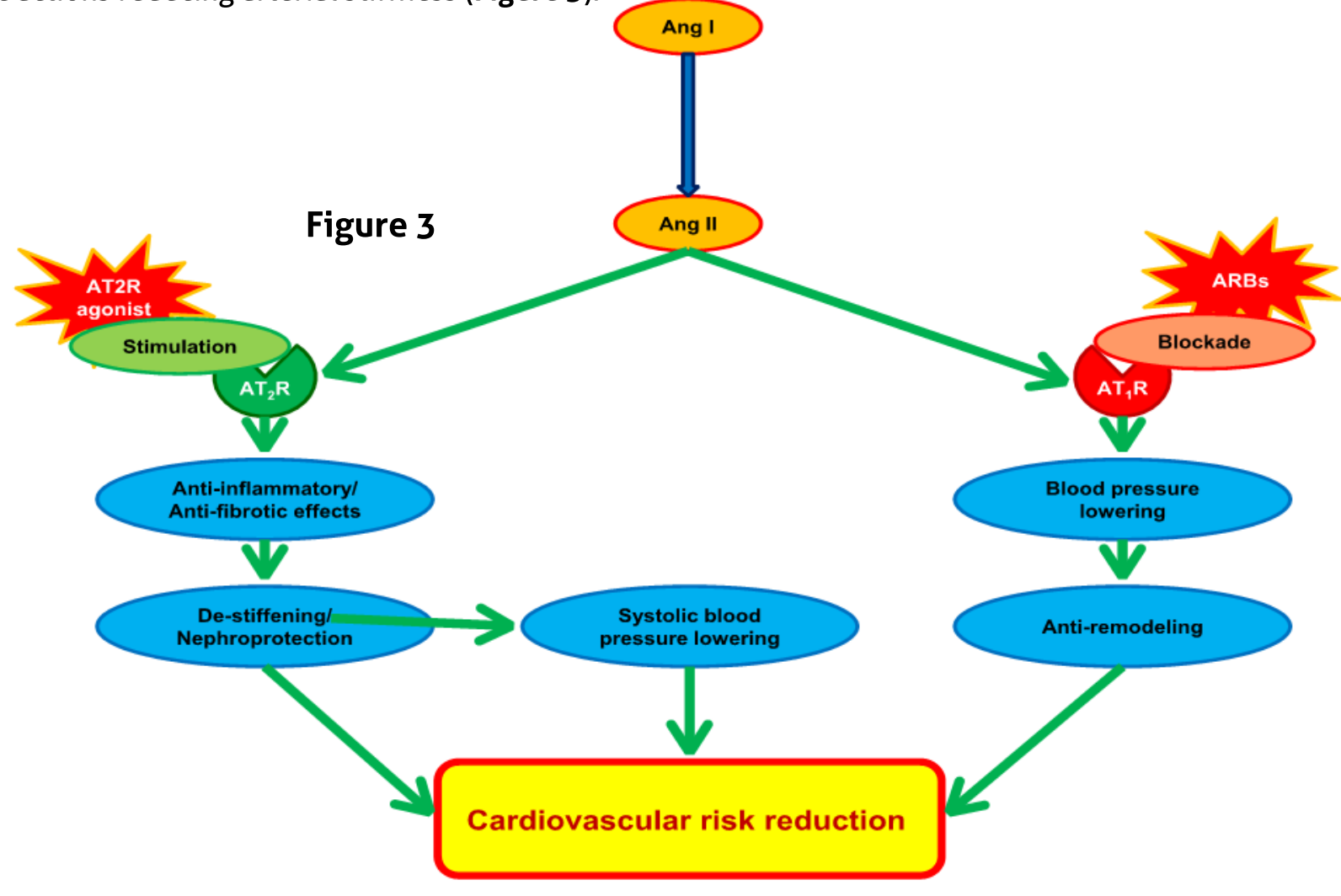

Combined angiotensin receptor modulation. Scheme showing the rationale for combining the stimulation of the AT2 receptor (AT2R) with the blockade of the angiotensin AT1 receptor AT1R by the AT1R blockers (ARBs). AT1R blockade lowers blood pressure directly and AT2R stimulation reduces arterial stiffness in the long term preventing the increase in the systolic blood pressure without compromising diastolic blood pressure values. Such a combined effect might be of particular significance in the elderly, in patients with isolated systolic hypertension or in end-stage renal patients. 


\section{FURTHER READING}

1. De Gasparo M, Catt KJ, Inagami T, Wright JW, Unger T. International union of pharmacology. XXIII. The angiotensin II receptors.Pharmacol Rev2000;52:415-72.PMID:10977869.

2. Guimond M-O, Gallo-Payet N. How does angiotensin AT(2) receptor activation help neuronal differentiation and improve neuronal pathological situations? Front Endocrinol2012;3:164. DOI: 10.3389/fendo.2012.00164.

3. Horiuchi M, Iwanami J, Mogi M. Regulation of angiotensin II receptors beyond the classical pathway. Clin Sci2012;123:193-203. DOI: 10.1042/CS20110677.

4. Kaschina E,NamsolleckP,Unger T. AT2 receptors in cardiovascular disease. Pharmacol Res. 2017 Nov;125(Pt A):39-47. DOI: 10.1016/j.phrs.2017.07.008.

5. Padia SH, Carey RM. AT2 receptors: beneficial counter-regulatory role in cardiovascular and renal function.Pflüg Arch Eur J Physiol2013;465:99-110. DOI: 10.1007/s00424-012-1146-3.

6. Paulis L, Foulquier $S$, Namsolleck P, Recarti C, Steckelings UM,Unger T. Combined angiotensin receptor modulation in the management of cardiometabolic disorders.Drugs. 2016 Jan;76(1):1-12. DOI: 10.1007/s40265- 015-0509-4.

7. Rodrigues-Ferreira S, Nahmias C. An ATIPical family of angiotensin II AT2 receptor-interacting proteins. Trends Endocrinol Metab TEM2010;21:684-90. DOI: 10.1016/j.tem.2010.08.009.

8. Unger T, Steckelings UM, Dos Santos, RAS, eds. The Protective Arm of the Renin Angiotensin System, Functional aspects and therapeutic implications.Academic Press, Elsevier 2015.ISBN: 978-0-12-801364-9.

9. Wan Y, Wallinder C, Plouffe B, Beaudry H, Mahalingam AK, Wu X, et al. Design, synthesis, and biological evaluation of the first selective nonpeptide AT2 receptor agonist.J Med Chem2004;47:5995-6008.PMID:15537354. DOI: $10.1021 /$ jm049715t

10. Willyard C. As drug target reemerges, the question is to block or stimulate it.Nature Med2014;20:222. DOI: $10.1038 / \mathrm{nm0314}-222$. 